\title{
Breaking the Cycle - Wege aus der starren gesellschaftlichen Konstruktion von Arbeit und Geschlecht in Europa
}

HEATHER HOFMEISTER und NADINE WITT

„People are border-crossers who make daily transitions between two worlds - the world of work and the world of family" (Campbell Clark 2000: 748). Diese Feststellung von Campbell Clark hebt die Vereinbarkeitsproblematik von Beruf und Familie im Leben von Männern und Frauen hervor, die oft unbemerkt, aber unzählige Male im Alltag auftritt.

Familie und Beruf sind die zentralen Lebensbereiche von Frauen und Männern in der heutigen europäischen Gesellschaft. Diese zwei Bereiche stehen in einer wechselseitigen, aber nicht gleichgewichtigen Beziehung. Im Folgenden wird diese Problematik detailliert aufgegriffen und mit zukunftsfähigen Handlungsempfehlungen verbunden. Wir beginnen mit einem kurzen historischen Überblick zu Familie und Beruf in Europa.

\section{Strukturelle Veränderungen von Familie und Beruf in Europa}

Seit der Industriellen Revolution wurde die außerhäusliche Aktivität des Mannes mit Beruf und bezahlter Arbeit gleichgesetzt. Häusliche Arbeit, die unbezahlt blieb, wurde mit der Rolle der Frau in Verbindung gebracht und erfuhr geringe Wertschätzung (vgl. Coontz 2005; vgl. Yalom 2001). Seit den 1940er Jahren ist eine Veränderung dieses Rollenkonzeptes wahrnehmbar (vgl. Coontz 2005; vgl. Yalom 2001). Die gesell- 
schaftliche Umstrukturierung der Industrieländer in der zweiten Hälfte des 20. Jahrhunderts führte zu deutlichen Veränderungen in der Struktur von Familien- und Berufsrollen, vor allem aber zu einer Umstrukturierung der Rolle der Frau in Beruf und Familie (vgl. Moen 1992; vgl. Ostner 1993). Diese Änderungen äußerten sich in der Pluralisierung der Lebensformen, z.B. im Rückgang von Eheschließungen, sowie im Anstieg von Zwei-Verdiener Haushalten (vgl. Edwards/Rothbard 2000; vgl. Quasier-Pohl 2001).

Mit der Integration der Frau in den Arbeitsmarkt und die dadurch erhöhte Erwerbsbeteiligung entstand die Problematik und Relevanz der Aufteilung unbezahlter Arbeit. Es wurde notwendig, die Familien- und Hausarbeit neu zu strukturieren (vgl. Hochschild 1989; vgl. Williams 2000; vgl. Coontz 2005). Die Doppelbelastung durch das gleichzeitige Versorgen einer Familie und die Ausübung eines Berufes ist eine „neuere" Diskussion, die seit ungefähr drei Jahrzehnten besteht (vgl. Schneewind 1999). Das System Familie und das System der bezahlten Arbeit müssen sich in der heutigen europäischen Gesellschaft anders organisieren als zu Zeiten, in denen die Frau die Rolle der Hausfrau inne hatte und sich um Hausarbeit, Kindererziehung und Pflege anderer Familienmitglieder kümmerte, während es die Rolle des Mannes war, seine Familie als Alleinverdiener finanziell zu versorgen (vgl. Hofmeister/ Blossfeld 2006; vgl. Moen 1992). Längere Ausbildungszeiten, ein durchschnittlich höheres Heirats- und Erstgebärendenalter und der Wunsch bzw. das Bedürfnis nach Karriere für beide Partner treffen auf eine (biologisch) begrenzte Phase der Familiengründung. Beruf, Karriere und Familiengründung treten zeitlich in Konkurrenz zueinander (vgl. Moen 1992). Die Herausforderung besteht darin, Familie und Beruf in Einklang zu bringen und Männer und Frauen nicht, z.B. auf Basis von traditionellen Geschlechterrollen, aus den für sie wichtigen Sphären auszugrenzen.

Dieses Kapitel wird einen historischen und gegenwärtigen Blick auf bezahlte und unbezahlte Arbeit in Europa werfen. Schwerpunkte stellen die Geschlechterunterschiede, Lebensverläufe und gesellschaftliche Strukturen dar, die einerseits Akteure beeinflussen, und andererseits von Akteuren beeinflusst werden. 


\section{Die Weiterentwicklung des Begriffs „bezahlte“ Arbeit}

Die gesellschaftliche Zuschreibung und Relevanz von bezahlter Arbeit durchlief seit dem Mittelalter bis zur heutigen Zeit drei Entwicklungsstufen (siehe Abb. 1):

Abb. 1: Trennung von privater und öffentlicher Sphäre in westlichen Ländern - ein Konstrukt der Industriellen Revolution

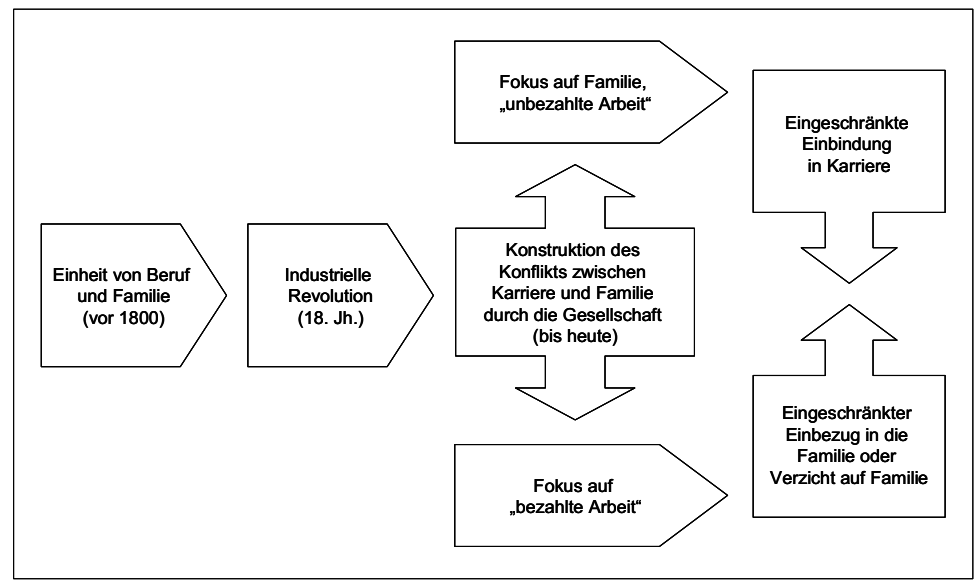

Im Mittelalter war die bezahlte Arbeit selbstverständlich. „Bezahlte“ und „unbezahlte“ Arbeit existierten im ,ganzen Haus“ nebeneinander. Knechte und Mägde erhielten Kost und Logis, ihnen wurde jedoch nicht zwingend Lohn gezahlt. Das bedeutet, dass Arbeit selbstverständlich, aber die „Bezahlung“ unterschiedlich war (Geld, Anrechte, Essen, Unterkunft, usw.). Mit der Industriellen Revolution wurden die zwei gegensätzlichen Sphären von Haus- und Erwerbsarbeit geschaffen. Die bezahlte Erwerbsarbeit wurde der unbezahlten häuslichen Arbeit übergeordnet und als wichtiger eingestuft. In der heutigen Gesellschaft wird Erwerbsarbeit genutzt, um die eigene Identität zu definieren (vgl. Baur 2001).

Im Mittelalter arbeiteten die meisten Menschen entweder in der Agrarwirtschaft oder in einem Familienunternehmen (vgl. Mills 1956). Vor der Industrialisierung stand anstelle der Trennung der öffentlichen und privaten Sphäre eine gewisse Einheit von Familie und Beruf (vgl. Raml 1993). Die Familienform des ,ganzen Hauses“ war gesellschaftlich normiert und folglich vorherrschend. Das ,ganze Haus“ war Produktionsstätte und Lebensbereich zugleich (vgl. Brüderl 1992; vgl. Mitterauer 1990). Dies bedeutete, dass nicht nur die Familienmitglieder, son- 
dern auch die Bedientesten wie Knechte, Gesellen und Lehrlinge diesem Hausverband zugehörten (vgl. Brüderl 1992; vgl. Mitterauer 1990). Der Lohn für ihre Arbeit bestand aus Nahrung und Unterkunft. Frauen waren nicht auf ein Arbeitsfeld beschränkt. Neben ihren Hausarbeitstätigkeiten, der Kindererziehung und Pflege anderer Familienangehöriger unterstützten sie ihren Ehemann bei der beruflichen Karriere. Die Familiengründung führte nicht zur Einschränkung ihrer Arbeitsbereiche. Frauen unterbrachen ihre Arbeit nur für die Zeit des Wochenbettes und nahmen im Anschluss ihre Tätigkeiten wieder auf. Erziehung und Versorgung wurden durch das Umfeld, d.h. durch Geschwister, Ältere usw. übernommen (vgl. Brüderl 1992; vgl. Mitterauer). Eine „Mehrfachbelastung“ und eine geringe Spezialisierung aller Familienmitglieder war ein integraler Bestandteil ihres Lebens zu dieser Zeit.

Der allgemeine Begriff „,bezahlte“ Arbeit verengte sich im 19. Jahrhundert zunehmend auf den Begriff der „Erwerbsarbeit“. Der Kapitalismus wurde in der westlichen Gesellschaft zum allgemeinen Prinzip des Wirtschaftens nach der Auflösung der feudal-ständischen Ordnung. Fortan wurde die bezahlte Arbeit zum Mittel von Tauschprozessen, zu einer Art Ware (vgl. Weber 1976; vgl. Marx 1867-94).

Eine Konsequenz der neuen industriellen fordistischen Arbeitsweise war die Auflösung der vorherrschenden Produktionsgemeinschaft, des „ganzen Hauses“ (vgl. Weber 1976). Die Einheit von Familie und Arbeitsstätte wurde aufgebrochen, Arbeits- und Wohnbereich wurden voneinander getrennt (vgl. Janas 2005; vgl. Bischoff 1994). Es kam zu einer sozial- und arbeitspolitisch regulierten Arbeitsteilung zwischen den zwei Sphären: (bezahlte) Erwerbsarbeit und häusliche (unbezahlte) Arbeit. Mit diesen zwei Arten von Arbeit wurden unterschiedliche Eigenschaften und Geschlechterrollen verbunden, die die täglichen Arbeiten von Mann und Frau und damit die Gestaltung ihrer Lebensverläufe trennten (vgl. Degler 1980). Beispielsweise waren berufstätige Frauen (aus der Mittelschicht) nach der Heimkehr von ihrer ,,bezahlten“ oder ,,produktiven“ Arbeit für die Hausarbeit zuständig. Dies verdeutlicht, dass es zu einer Rollenaufteilung im Haushaltsbereich kam (vgl. Voß/Jürgens 2007). Diese Trennung wurde nicht nur im sozialen, sondern auch im politischen Bereich vollzogen: dies geschah in Form von Gesetzen, z.B. über das Arbeitsverhältnis, die Ehe und sogar im aufkommenden Bereich der Wirtschaft, wie Folbre und Abel (1989) bereits in ihrer Studie über die USA erklärten: „The census institutionalized a definition of work as market work that literally devalued women's unpaid work" (547).

Die Erwartungen an die unbezahlte Arbeit von Frauen stiegen enorm an: Sauberkeitsstandards, Aufgaben im Haushalt, Erwartungen an Kin- 
dererziehung und soziale Unterhaltung erhöhten sich. Durch die technischen Entwicklungen, die mehr Arbeit in kürzerer Zeit ermöglichten, wurde das Arbeitspensum der Mittelklasse-Frau um ein Vielfaches erhöht (vgl. Cowan 1941). Die neuen Haushaltsprodukte und Technologien ermöglichten aber auch eine stärkere Integration in den Arbeitsmarkt, da sie die Tätigkeit der Frauen im Haushalt erleichterten (vgl. Cohen 1998) und ihnen so mehr „Zeit“ für die bezahlte Erwerbsarbeit gewährten.

Im Laufe des 20. Jahrhunderts änderte sich die Einstellung der Frau zur Erwerbsarbeit (vgl. Ostner 1993). Die seit der Nachkriegszeit vorherrschende traditionelle Familienordnung wurde von der Idee der Vereinbarkeit von Beruf und Familie abgelöst, welche gleichzeitig das Alleinverdienermodell kritisierte (vgl. Jürgens/Voß 2007). Für den Menschen in der heutigen westlichen Gesellschaft hat der Beruf neben sozialen, kulturellen, politischen und rechtlichen auch eine personale Bedeutung (vgl. Lachmann 1995). Erwerbsarbeit wird benutzt, um die eigene Identität und den Selbstwert zu konstruieren. Bezahlte Arbeit hat folglich einerseits den Zweck, Lebensunterhalt und Sicherheit zu gewährleisten, andererseits aber auch einen ideellen Stellenwert für Mann und Frau, indem sie Selbstachtung, Achtung durch andere erfahren und das Gefühl bekommen, kreativ und produktiv zu sein (vgl. Lachmann 1995; vgl. Kolinsky 1995).

\section{„Bezahlte“ und „unbezahlte“ Arbeit}

Was ist unter „,bezahlter“ und „unbezahlter“ Arbeit zu verstehen? Eine Neubesetzung des Begriffs „Arbeit“ setzt eine Abwendung vom Modell der zwei Sphären, der öffentlichen und der private Sphäre, voraus. Die öffentliche Sphäre basiert auf Erwerbsarbeit, Lohn und Ansehen, die private auf Familien- und Hausarbeit, die gewöhnlich von Frauen verrichtet wird. In vielen Studien wurde herausgestellt, dass die vorrangig von Frauen geleistete häusliche und familiäre Arbeit meist wenig Wertschätzung erhält und die erfolgte Leistung somit „unsichtbar“ bleibt (vgl. Folbre/Abel 1989). „Sichtbare“ Arbeit in diesem Sinne fokussiert sich auf bezahlte Arbeit und ignoriert gleichzeitig andere Formen von Arbeit, z.B. die unbezahlte Hausarbeit und Kindererziehung, sowie die Pflege von Familienmitgliedern. Wir ziehen die Begriffe „bezahlte“ und „unbezahlte“ Arbeit vor. „Bezahlte“ Arbeit meint Erwerbsarbeit (auf dem Arbeitsmarkt); „unbezahlte“ Arbeit meint unentgeltliche Ausführung von Hausarbeit, Kindererziehung und Pflege von Familienmitgliedern. 
Die Unterscheidung zwischen ,privater“ und „öffentlicher“ Sphäre lehnen wir aus folgenden Gründen ab: (1) „Unbezahlte“ Arbeit betrifft die ganze Bevölkerung, beispielsweise die Erziehung von Kindern. Folglich ist ihre Auswirkung nicht auf die private Sphäre begrenzt und greift in die öffentliche Sphäre ein. (2) Es ist richtig, dass „,bezahlte“ Arbeit zum Teil in der öffentlichen Sphäre stattfindet. Die Erzeugnisse dieser Arbeit können jedoch als teilweise privat bezeichnet werden, da die Identität eines Menschen in sie eingeht. (3) Neue Arbeitsmodelle ermöglichen es Arbeitnehmern zunehmend, von zu Hause aus zu arbeiten, über Mobiltelefon und Internet können Arbeitgeber und Arbeitnehmer überall in Verbindung stehen. Dies führt zu einer Vermischung von privater und öffentlicher Sphäre. Folglich ist diese Unterscheidung nicht länger haltbar.

Die Assoziationen, die mit dem Begriff „Arbeit“ in der europäischen Gesellschaft verknüpft sind, müssen sich in naher Zukunft ändern, damit Erwerbsarbeit und häusliche Arbeit als gleichwertig anerkannt werden können. Dies könnte bewirken, dass die häusliche Arbeit nicht länger geringer wertgeschätzt wird als die bezahlte Arbeit. Durch die gesellschaftliche Akzeptanz und Wertschätzung könnte die unbezahlte Arbeit für den Mann attraktiver werden und von ihm im höheren Maße (als gegenwärtig) übernommen werden.

\section{Der Mythos der Karriere - oder: Warum werden alte Konzepte der Arbeitsteilung zum Problem?}

Es soll aufgezeigt werden, wie die Ausbildung von Geschlechterrollenzuweisungen, die in der heutigen westlichen Gesellschaft Barrieren für die Vereinbarkeit von Familie und Beruf darstellen, einerseits zu einer doppelten Belastung der Frau und andererseits zur Ausgrenzung des Mannes aus seiner Familie durch mangelnde gesellschaftliche Akzeptanz des fürsorglichen teilzeitbeschäftigten Vaters oder des Hausmannes führt.

Einstellungen zu Geschlechterrollen beziehen sich auf Erwartungen in Bezug auf Verhalten, Verantwortung und Aktivitäten, die Frauen bzw. Männern angemessen sind (vgl. Eagly 1987). Männer und Frauen haben jedoch unterschiedliche Ansichten, was angemessene Rollen und Verhaltensregeln in Bezug auf bezahlte und unbezahlte Arbeit betrifft. Individuen mit einer traditionellen Einstellung halten es für richtig, dass es die Rolle einer Frau ist, Hausfrau und Mutter zu sein, und die Rolle des Mannes darin besteht, der Ernährer der Familie zu sein. Egalitär eingestellte Menschen halten es für richtig, dass es die Rolle von Frau und 
Mann sein sollte, die Familie nicht nur finanziell zu unterstützen, sondern sich auch an Hausarbeit und Kindererziehung und an anderen als feminin angesehene Bereichen, wie z.B. Kochen, Einkaufen, Waschen, Bügeln, zu beteiligen. Im Allgemeinen lässt sich feststellen, dass die Ungleichheit in der Aufteilung der bezahlten und unbezahlten Arbeit zwar abgenommen hat, aber im Kern bestehen geblieben und größtenteils auch nach wie vor geschlechtsspezifisch verteilt ist (vgl. Bianchi et al. 2000).

Die Industrielle Revolution hat tiefgreifende Veränderungen innerhalb der Familie und der Arbeitswelt hervorgerufen (vgl. Moen 1992). Geschlechtsstereotype, die teilweise noch in der heutigen Gesellschaft bestehen, bildeten sich zu dieser Zeit heraus. Sie haben Einfluss auf den weiblichen und männlichen Lebensweg in der heutigen europäischen Gesellschaft und sind Ursache für psychische und strukturelle Barrieren bei der Vereinbarkeit von bezahlter und unbezahlter Arbeit. Zugleich aber wird das alte Konzept der Arbeitsteilung, d.h. die Trennung in häusliche, unbezahlte Arbeit und außerhäusliche, bezahlte Arbeit gegenwärtig für viele Männer und Frauen in Europa zum Problem (vgl. Moen 2001).

Die Umsetzung von Geschlechterrollen führen auf der Individualebene von Mann und Frau zu einem normierten Umgang mit Familie und Beruf. Machtbeziehungen, die weibliche Unterordnung und männliche Dominanz verlangen und zu einer geschlechtstypischen Aufteilung von häuslicher Arbeit und Erwerbsarbeit führen, können auf die soziale Komponente des Geschlechts zurückgeführt werden (vgl. Connell 1999). Die Gesellschaft achtet, basierend auf Normen und Werten, auf die Hierarchie zwischen Mann und Frau (vgl. Connell 1999). Diese polaren Zuschreibungen, die sich komplementär ergänzen, vertiefen die Gegensätzlichkeit von bezahlter und unbezahlter Arbeit. Die Idee des männlichen Alleinverdieners und der weiblichen Hausfrau etablierte und verfestigte sich. Das Lohnsystem unterstützt ebenfalls die Gegensätzlichkeit von Produktions- und Reproduktionsbereich. An diese Unterscheidung sind kulturelle Werte und Wertschätzungen geknüpft, die zu einer Aufwertung der bezahlten Erwerbsarbeit und gleichzeitig zu einer Abwertung oder Geringschätzung häuslicher und familiärer Arbeit führen (vgl. Connell 1999). Die bezahlte Erwerbsarbeit wird als wichtig und mit Prestige verbunden definiert, während die unbezahlte Haus- und Familienarbeit nicht im Sinne von „Arbeit“, welche gesellschaftlichen Nutzen bringt, wahrgenommen wird. Dadurch entstehen Beschreibungen oder Erklärungen dafür, daß Männer sich nicht um die Kindererziehung kümmern können, wie z.B. die folgende Rechtfertigung aus einer Diskussion unter Männern über Geschlechterrollen zeigt: 
„Außerdem ist's halt noch schwierig für einen Mann von der Nachbarin gefragt zu werden: ,Na, Herr Müller, heute wieder ein Kessel Buntes?' Oder mit der Tüte Pampers angetroffen und gefragt zu werden: „Na, Herr Müller, ist ihre Jüngste noch nicht aus den Windeln?“ (Witt 2008).

Diese kurzen Ausführungen zeigen, dass das vorherrschende traditionelle Arbeitsteilungskonzept den Bedürfnissen der heutigen europäischen Gesellschaft nicht mehr entspricht. Dies wird im Folgenden detaillierter erläutert.

\section{Was bedeutet es, in der heutigen Zeit in Europa eine Frau zu sein?}

Eine erhöhte Integration in den Arbeitsmarkt, traditionelle Geschlechterrollenzuschreibungen sowie Diskriminierungen bewirken, daß es für die Frauen eher schwieriger geworden ist, Familien- und Erwerbsarbeit miteinander zu vereinbaren.

Die traditionelle Ansicht über die Arbeitsteilung zwischen bezahlter und unbezahlter Arbeit ist nirgendwo in Europa gänzlich verschwunden (vgl. Lück 2006), auch wenn sie sich in weiten Teilen Europas positiv verändert, d.h. etwas egalisiert, hat (vgl. Bolzendahl/Myers 2004). Die Geburt des ersten Kindes führt oft zu einer Traditionalisierung der Geschlechterrollen (vgl. Becker/Moen 1999; Reichart 2007; Röhler 2000; Fux 2002). Nachweislich kümmern sich mehr Frauen als Männer um den Haushalt und die Kindererziehung (vgl. Lück 2006; vgl. Coltrane 2000). Auch in den nordeuropäischen Ländern, in denen die formale Teilnahme der Frau am Arbeitsmarkt am höchsten ist, nehmen Frauen $\mathrm{zu}$ einem höheren Anteil als Männer Erziehungsurlaub (vgl. Orloff 2002). Es ist erwiesen, dass Ehe und Kinder oft zu einem Anstieg von Hausarbeit bei Frauen und gleichzeitig zu einer geringeren Beteiligung am Haushalt bei Männern führen (vgl. Coltrane 2000; vgl. Kaufmann/Uhlenberg 2000; vgl. Greenstein 1996). Die höhere Verantwortung der Frauen für Kindererziehung und Hausarbeit erschwert ihnen die Vereinbarkeit von Erziehung, Haushalt und bezahlter Arbeit und wird von ihnen als störender Einschnitt, als Sanktion, empfunden (vgl. Gershuny 1996; Notz 1991) (siehe Abb. 2). 
Abb. 2: Konzeptioneller Teufelskreis für die Arbeit von Frauen

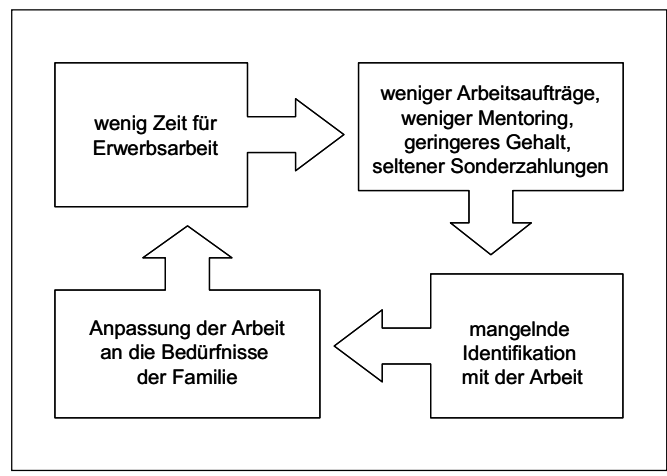

Im Blick auf die kulturellen Bedingungen für die Vereinbarkeit von Beruf und Familie benennt Pfau-Effinger (2001; 2003; 2004) zwei Entwicklungen: die erste nennt sie die „Modernisierung der männlichen Versorgerehe“, die zweite das „Doppelversorgermodell“. Das erste Modell, die Versorgerehe, herrscht in den Niederlanden, Großbritannien und Deutschland vor. Hier wird durch politische Maßnahmen, wie mangelnde Kleinkinderbetreuungsmöglichkeiten und steuerliche Vergünstigungen, den Müttern nahe gelegt, sich vor allem in den ersten Lebensjahren der Erziehung ihrer Kinder zu widmen. Geringfügige Beschäftigung oder eine Teilzeitbeschäftigung der Mutter werden toleriert, was aber die finanzielle Abhängigkeit der Frau vom Mann nicht aufhebt und eine Reduzierung der Erwerbsarbeit bedeutet (vgl. Pfau-Effinger 2001; vgl. Lück 2006). Das zweite Modell, das Doppelversorgermodell - vorherrschend in skandinavischen Ländern und Frankreich - basiert auf der Grundlage, dass sowohl Männer als auch Frauen einer Vollzeitbeschäftigung nachgehen dürfen und sollen, auch wenn Kinder vorhanden sind (vgl. Pfau-Effinger 2001). Kinderbetreuung wird nicht als private, auf die Familie begrenzte Aufgabe angesehen, sondern als Verantwortungsbereich der gesamten Gesellschaft. Das Doppelversorgermodell hat zur Folge, dass Frauen eine überdurchschnittlich hohe Erwerbstätigenquote aufweisen. Eine Änderung der Aufteilung von Arbeit im privaten Bereich, d.h. eine erhöhte Unterstützung bei der Hausarbeit durch den Mann kann laut Pfau-Effinger (2001) trotzdem nicht festgestellt werden.

Mit diesen theoretischen Modellen sind die unterschiedlichen Erwerbsquoten von Frauen in den europäischen Ländern erklärbar. In manchen europäischen Ländern nähert sich die Anzahl der Arbeitnehmerinnen denen der Arbeitnehmer an. Mehr Frauen als zuvor lehnen die Ausübung der traditionellen Rolle ab, um eine Karriere verfolgen zu können - eine berufsorientierte Haltung, die zuvor als typisch männlich 
galt (vgl. Goldin 2004; vgl. Orloff 2002). Immer mehr verheiratete Frauen sind erwerbstätig und tragen zu einem größeren Teil zum Unterhalt bei als in der Vergangenheit (vgl. Raley et al. 2006). „Sozialdemokratische" Länder wie Schweden und Dänemark weisen eine hohe und weiterhin ansteigende Frauenerwerbsquote auf; diese Entwicklung trifft ebenfalls auf die „liberalen“ Länder wie Großbritannien zu. „Konservative Staaten“"wie Deutschland weisen ebenfalls einen Anstieg der Frauenerwerbsquote auf, diese ist jedoch geringer als in den liberalen Ländern. Die niedrigste Anzahl von Frauen im Erwerbsleben können in den „familienorientierten“ Ländern wie Italien und Spanien verzeichnet werden (vgl. Hofäcker 2006).

Diese Verschiebung von ,unbezahlter“ Arbeit in Richtung ,bezahlte" Arbeit, wenn auch europaweit in unterschiedlichem Ausmaß, ist von fundamentaler Bedeutung (vgl. Folbre/Nelson 2000). Obwohl ein Anstieg der Frauenerwerbsquote in den europäischen Ländern zu verzeichnen ist, ist jedoch der typische Arbeitnehmer nach den Vorstellungen des Arbeitgebers immer noch männlich und ungebunden (vgl. OECD 2008). Wir illustrieren diesen Teufelskreis in Abbildung 3.

Abb. 3: Kreislauf von Faktoren, die die geringere Einbindung von Frauen in den Arbeitsmarkt beeinflussen

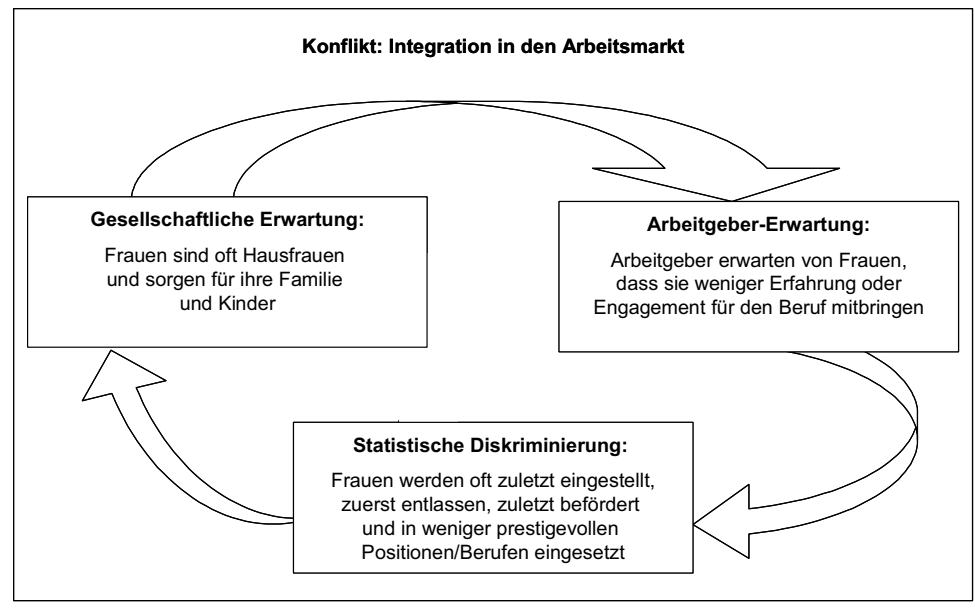

Diese Diskriminierung auf dem Arbeitsmarkt führt dazu, dass Männer bevorzugt eingestellt werden: die Wahrscheinlichkeit einer Erwerbsarbeit nachzugehen ist für europäische Frauen 20\% geringer als für Männer, ihr Gehalt für die Ausübung der gleichen Tätigkeit ist $17 \%$ niedriger (vgl. OECD 2008). Die Anzahl der Frauen, die gleiches Gehalt für die gleiche Tätigkeit erhalten, ist vergleichsweise gering (vgl. OECD 2008; 
vgl. Bielby/Baron 1986; vgl. Hersch/Stratton 2002). 8\% der Beschäftigungsunterschiede und 30\% der Lohnunterschiede zwischen Mann und Frau können auf diese Diskriminierung am Arbeitsplatz zurückgeführt werden (vgl. OECD 2008).

Das Konzept der Diskriminierung bietet Erklärungsmöglichkeiten für die Bevorzugung eines Mannes bei der Neueinstellung, auch wenn die Frau vergleichbare Eigenschaften und Fähigkeiten aufweist (vgl. Bielby/Baron 1986; vgl. Becker 1971; vgl. Blau 1984). Nach diesem Modell lässt sich aufzeigen, dass Arbeitgeber dazu neigen, die bezahlte Arbeitsproduktivität von Männern und Frauen (für die Erfüllung der gleichen Aufgabe) unterschiedlich zu bewerten. Viele Arbeitgeber bevorzugen beispielsweise kinderlose Frauen und sind davon überzeugt, dass Frauen sich aus bezahlter Arbeit zurückziehen oder teilzeitbeschäftigt sein sollten, wenn sie zur Mutter werden oder junge Kinder haben (vgl. Treas/Widmer 2000). Wenn dem Arbeitgeber durch die Kündigung einer Angestellten und dem daraus folgenden Einstellen und Einarbeiten eines neuen Arbeitnehmers zu hohe Kosten entstehen, ist es wahrscheinlich, dass er zukünftig männliche Arbeitnehmer bevorzugen wird. Vor allem Mütter sind es, die auf dem Arbeitsmarkt benachteiligt werden. Mutterschaft scheint das größte Hindernis auf dem Weg zur ökonomischen Gleichstellung von Männern und Frauen zu sein (vgl. Crittenden 2001). Profitmaximierende Unternehmen reservieren Arbeitsplätze für die Gruppe von Arbeitnehmern, von der eine höhere Produktivität zu erwarten ist (vgl. Bielby/Baron 1986). Ist es dem Arbeitgeber im Einzelfall nicht möglich herauszufinden, ob z.B. eine Frau weniger (als ein Mann) dazu neigt, den Arbeitsplatz zu kündigen oder weniger psychologisch in diesen involviert zu sein, dann wird er einen männlichen Arbeitnehmer bevorzugen, Frauen werden vermehrt in Positionen eingesetzt, in denen die wirtschaftlichen Kosten durch Ausfall (Kündigung) niedrig sind. Wenige Arbeitgeber wissen, dass Männer aus Krankheitsgründen durchschnittlich öfter ausfallen als Frauen mit Kindern (vgl. Bielby/Baron 1986). Dieses Faktum hat jedoch keine negativen Auswirkungen auf die Karriere von Männern. Mangelnde Informationen (auf Seiten des Arbeitgebers) führen also zur Bevorzugung des männlichen Geschlechts bei einer Neueinstellung (vgl. Bielby/Baron 1986).

Dies führt dazu, dass Frauen zwei Arten von Kompromissen schließen (müssen): einerseits gibt es Frauen, die sich gegen eine Karriere und für die Familie entscheiden. Andererseits gibt es Frauen, die eine starke Einbindung in eine Familie vermeiden, indem sie entweder alleinstehend bleiben oder sich gegen eigene Kinder entscheiden. Die sich widersprechenden Rollen von Beruf und Familie fordern von Frauen eine Ent- 
scheidung; sie müssen wählen, welche Rolle für ihr Leben wichtiger sein soll (vgl. Friedman/Greenhaus 2000).

Die bisherige Darstellung zeigt, dass Gleichheit von Männern und Frauen im Erwerbsleben nicht allein von Zugangschancen, Ausbildung und gleichen Voraussetzungen auf dem Arbeitsmarkt abhängig ist, sondern auch von der Familiengründung und Kinderbetreuung. Die heutige Gesellschaft ist noch nicht dazu bereit, die Hausarbeit als „unbezahlten“ Arbeitsplatz für die Frau aufzugeben. Hinzu kommt, dass es keinen anderen Arbeitsbereich gibt, der von Frauen (ungewollt) dominiert wird und zudem unbezahlt ist. Hausarbeit und Kindererziehung erhalten niedrige öffentliche Wertschätzung, da nur „bezahlte“ Arbeit als „richtige“ Arbeit gesellschaftlich anerkannt wird.

Es stellt sich die Frage, wie Frauen es schaffen können, Karriere und Familie zu integrieren, oder anders: wie sie ein erfolgreiches und zufriedenes Leben nach ihren Vorstellungen leben können.

\section{Was bedeutet es, in der heutigen Zeit in Europa ein Mann zu sein?}

Die Rolle des Mannes hat sich seit dem 18. Jahrhundert mehrfach gewandelt (vgl. Baur/Hofmeister 2008). Im 18. Jahrhundert definierte sich der Mann über seine gestaltende und dominante Rolle und Position innerhalb der Gesellschaft (vgl. Hendrix 1995; vgl. Zook 2002). Werte und soziale Strukturen wurden über die Heirat und die Institution der Ehe aufrecht erhalten (vgl. Baur/Hofmeister 2008). Der (verheiratete) Mann war nicht nur für seine Familie, sondern auch für das ,gesamte Haus“ verantwortlich, zu dem auch Diener, Knechte und Mägde, uneheliche und Waisenkinder gehörten (vgl. Baur/Hofmeister 2008; vgl. Zook 2002). Die Verantwortung der Kindererziehung und der Ausbildung der Söhne lag demnach in den Händen des Mannes. Väter wurden als warmherzig angesehen, verbrachten viel Zeit mit ihrer Familie, mit Liebe und Stolz; Frauen hingegen wurden als unfähig angesehen, Verantwortung zu übernehmen und wahre Gefühle empfinden zu können (vgl. Baur/Hofmeister 2008).

Die Rolle des Mannes änderte sich wieder mit der Industriellen Revolution. Arbeitsplatz und Wohnort wurden voneinander getrennt, das „ganze Haus“ folglich aufgelöst (vgl. Kocka 1990). Die Aufgabe des Mannes war es nun (und ist es in Teilen Europas noch heute), Beschützer, Ernährer und Erzeuger zu sein. Folglich war er für das finanzielle Auskommen der Familie verantwortlich und musste einer Einkommen 
abwerfenden „Erwerbsarbeit“ nachgehen, während die Frau sich um Kinder und Haushalt, also um unbezahlte, häusliche Tätigkeiten kümmerte. Die unbezahlte Hausfrau wurde zum Prestigeobjekt (vgl. Niehuss 1999). $\mathrm{Zu}$ dieser Zeit wurde die Verantwortung für Kindererziehung vom Mann auf die Frau übertragen (vgl. Schütze 1988). Fortan definierte sich der Mann über den Erfolg seines Berufes (vgl. Trepp 1996; vgl. Schütze 1988; vgl. Rotundo 1993).

Diese Vorstellung von Männlichkeit basiert auf der Normvorstellung der bürgerlichen Familie. Für den traditionellen Mann in der heutigen europäischen Gesellschaft ist die Aufteilung der Bereiche Familie und Erwerbsarbeit fest vorgeschrieben; auch wenn die Frau erwerbstätig ist, wird dieses Bild vom traditionellen Alleinverdiener nicht angetastet, da das Gehalt als Zusatzeinkommen verstanden wird (vgl. Puchert/Höyng 2008). Der Mann konzentriert sich auf seine Ernährerrolle und dadurch auf seine Erwerbstätigkeit. Indem er für das Familieneinkommen sorgt, versteht er seine bezahlte Arbeit als einen essentiellen Beitrag für die Familie, der es ihm ermöglicht, sich der Hausarbeit und Kindererziehung völlig zu entziehen (vgl. Puchert/Höyng 2008).

Kimmel (1987) gibt einen Überblick darüber, wie sich die Definition von Männlichkeit seit den 1950ern verändert hat. Er zeigt, dass Männer sich „,neu definieren“ müssen, weil Frauen sich und ihre Geschlechterrollen ändern. Frauen in der heutigen Zeit möchten immer häufiger beides: Kinder und Karriere. Folglich müssen Männer bzw. Väter dies auch wollen. Auch wenn Männer vorgeben, dies zu befürworten, tritt eine Veränderung in der Realität nur selten ein. Laut Kimmel möchte nur eine Minderheit der Männer die Position mit ihren Frauen tauschen. Männer ziehen der Hausarbeit und Kindererziehung bezahlte, ,richtige“ Arbeit vor. Für Männer stellt die Beteiligung an der Hausarbeit bereits ein Kompromiss dar. Kimmel schreibt weiter, dass die Definition von Männlichkeit bemerkenswert stabil bleibt. Auch wenn es die ersten „,neuen Väter“ gibt, die sich zusammen mit ihrer Frau um die Kindererziehung kümmern, so stellt diese Gruppe von Männern noch immer eine Minderheit dar. Männer werden nach wie vor, auch wenn es eine neue Generation von Männern gibt, die sich an Kindererziehung und Haushalt beteiligen, am Erfolg im Beruf in der öffentlichen und nicht der privaten Sphäre gemessen (vgl. Volz 2007) (siehe Abb. 4).

Dies erklärt, warum die Vereinbarkeit von Beruf und Familie im überwiegenden Maße als ein weibliches Phänomen oder Problem wahrgenommen wird. Auch in der Politik zur Gleichstellung der Geschlechter wird das Vereinbarkeitsproblem von Beruf und Familie weitgehend mit einem weiblichen Problem und folglich mit einer Frauenförderung gleichgesetzt (Puchert 2008). 
Abb. 4: Konzeptionelles Modell, das den Kreislauf der mangelnden Integration von Männern in das Familienleben beschreibt

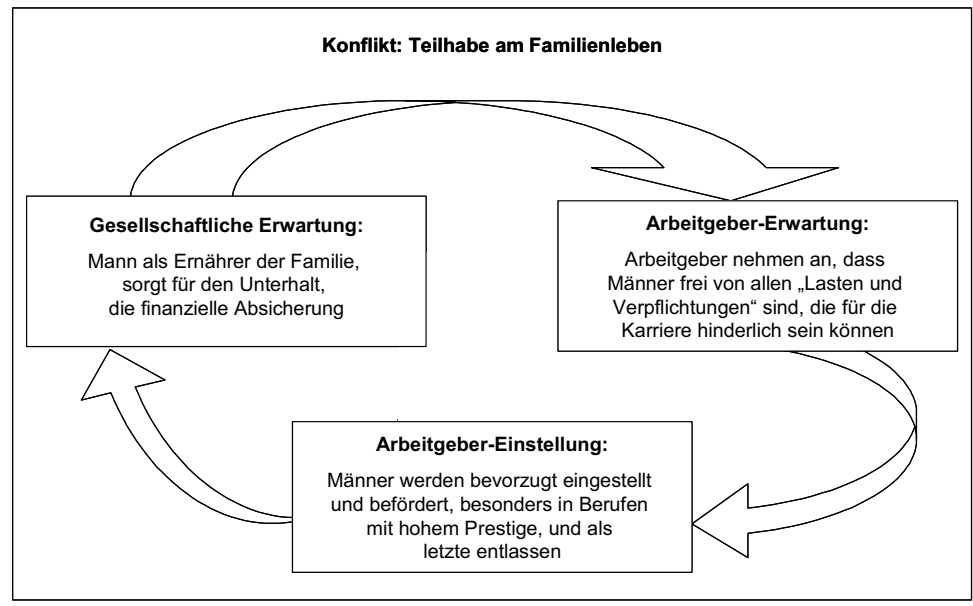

Nach Astrachans (1992) Definition einer neuen Männlichkeit hat der „neue Mann“ seine traditionelle Geschlechterrolle größtenteils überwunden, ebenso den Versuch, Macht monopolisieren zu wollen. Der Mann besteht nicht mehr darauf, der alleinige Ernährer der Familie zu sein und weigert sich, Erwerbsarbeit als Lebensziel zu begreifen. Der neue Mann ist gefühlvoll, kann über Probleme und Schwächen reden, und die Frau bei der Umsetzung von Gleichheit und Unabhängigkeit unterstützen. Er nimmt seinen Beruf nicht wichtiger als seine Familie und übernimmt die Hälfte der Verantwortung für Hausarbeit und Kindererziehung (vgl. ebd.). Der „neue Mann“ fordert eine erhöhte Teilnahme am Familienleben und hinterfragt den einseitigen Schwerpunkt auf dem Berufsleben. Demzufolge ist eine Balance zwischen Beruf und Familie für die Erfüllung von persönlichen Bedürfnissen auch für den Mann relevant. Sehr langsam wird der aktive Vater in unserer Gesellschaft akzeptiert, der sich beruflich zurücknimmt, um für seine Familie sorgen zu können (vgl. Ranson 2001; vgl. Astrachan 1992: 360) (siehe Abb. 5).

Väter, die Erziehungsurlaub beanspruchen oder einer Teilzeitbeschäftigung nachgehen wollen, um sich stärker in ihr Familienleben einbinden zu können, stellen die Minderheit in der heutigen europäischen Gesellschaft dar und sind somit „Vorreiter“ für diesen Lebensstil (vgl. Gräfinger 2001; vgl. Brayfield 1995). Ihr Verständnis von der Verteilung von Haus- und Familienarbeit und Erwerbstätigkeit verändert tradierte Vorstellungen und Rollenkonzepte, die traditionell einem Mann zugeschrieben werden. Dass diese „,neuen“ Männer privaten, gesell- 
schaftlichen und beruflichen Hindernissen ausgesetzt sind, lässt Rückschlüsse auf das vorherrschende Männerideal zu (vgl. Gräfinger 2001).

Abb. 5: Entwicklung der Vereinbarkeitsproblematik des Mannes

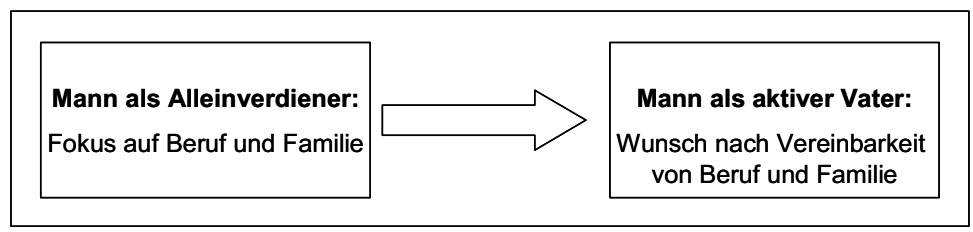

Einem Wertewandel tradierter Geschlechterrollenbilder stehen massive Hindernisse entgegen. Männer werden an einem Abweichen vom tradierten Rollenbild, aufbauend auf einer stark leistungs- und wettbewerbsorientierten Gesellschaft und Sozialisation, gehindert (vgl. Gesterkamp 2005). Weichen Männer von dem Ideal des Ernährers ab, müssen sie mit Barrieren am Arbeitsplatz, einem Stillstand der Karriere und mit Vorurteilen im Umfeld, dem Freundeskreis, der Familie und der weiteren Gesellschaft, rechnen. Mitarbeit bei der Erziehung und im Haushalt wird nicht als eigenes Interesse des Mannes identifiziert, sondern als Entlastung der Frau durch den Mann (vgl. ebd.; Mühling/Rost 2007).

Für die Frau äußert sich die Problematik der Vereinbarkeit vor allem in Form eines Zeit- und Koordinierungsproblems sowie in Karriere- und Gehaltseinbußen. Zusätzlich zu diesen Problemen liegt die Vereinbarkeitsproblematik für den Mann im traditionellen Rollenbild, welches Männlichkeit über beruflichen Erfolg oder Erwerbsarbeit definiert. Folglich scheint ein Gleichgewicht von bezahlter und unbezahlter Arbeit für den Mann ein schwer erreichbares Ziel zu sein, eine Berufspause oder Teilzeitbeschäftigung schwer durchsetzbar (vgl. Arn/Walter 2004; vgl. Gesterkamp 2005).

$\mathrm{Zu}$ diesem Hindernis, das durch traditionelle Rollenvorstellungen hervorgerufen wird, treten betriebliche Hindernisse. Väter müssen sich mit betrieblichen Hindernissen auseinandersetzen, wenn sie sich verstärkt in das Familienleben einbinden möchten, um ihre Kinder zu erziehen und aufwachsen zu sehen (vgl. Gersterkamp 2005). Die Verweigerung von Überstunden oder die Forderung nach Teilzeitbeschäftigung werden durch den Arbeitgeber oft als Arbeitsverweigerung gewertet, die in seltenen Fällen auch mit einer Kündigung einher gehen kann (vgl. Vaskovics/Rost 1999). Es scheint, dass Arbeitgeber den „,neuen Vater“ nicht akzeptieren und am alten, traditionellen Rollenideal festhalten (vgl. Werneck 1998; vgl. Peinelt-Jordan 1996). Es stellt sich für den „,neuen Mann“ als schwierig dar, abweichendes Verhalten zu zeigen und sich 
gegen das traditionelle Rollenbild zu stellen (vgl. Gesterkamp 2005; vgl. Schnack/Gesterkamp 1998).

Folglich ist die Frage gerechtfertigt, was es bedeutet, ein Mann in der heutigen Gesellschaft zu sein. Es scheint, dass der „neue“ Mann nicht mehr Alleinernährer der Familie ist, aber auch nicht der alleinige Erzieher seiner Kinder, sondern eine Kombination aus beidem. Neben seiner ,bezahlten“ Berufstätigkeit widmet er sich folglich der „unbezahlten“ Kindererziehung und Hausarbeit. Dies zeigt, dass der moderne Mann der Doppelbelastung von Beruf und Familie mit ähnlichen Konsequenzen ausgesetzt ist, wie dies bei den Frauen der Fall ist. Bei Männern, deren Identität und Definition von Männlichkeit historisch stark durch Arbeit und nicht durch Kindererziehung und Hausarbeit geprägt wurde, ist deshalb die Wahrscheinlichkeit für Kinderlosigkeit hoch. Dies löst jedoch nicht die Vereinbarkeitsproblematik zur Zufriedenheit von Männern und Frauen und erhöht die Wahrscheinlichkeit für eine Kinderlosigkeit vieler Paare.

\section{Handlungsempfehlungen}

Die Bedürfnisse von Männern und Frauen in der heutigen westlichen Gesellschaft haben sich verändert. Sowohl Männer als auch Frauen suchen nach neuen Wegen, die eine Vereinbarkeit von Familie und Beruf ermöglichen. Zusätzlich haben sich die Bedürfnisse der westlichen Gesellschaft verändert (vgl. Moen 2001; Williams 2000). Nicht nur das Talent von Männern, sondern auch das von Frauen muss für produktive Tätigkeiten genutzt werden; die wirtschaftliche Lage und der erhöhte Anteil an älteren Menschen in den Industrieländern erfordert, dass weder Männer noch Frauen ein Vereinbarkeitsproblem von bezahlter und unbezahlter Arbeit haben dürfen. Das Konzept des männlichen Alleinverdieners und der weiblichen Hausfrau ist in der heutigen Zeit auch aus ökonomischen Gründen nicht mehr haltbar (vgl. Moen 2001).

Wie zuvor aufgezeigt, hat die traditionelle Rollenzuschreibung in der heutigen Gesellschaft negative Konsequenzen für Mann und Frau. Hierbei handelt es sich einerseits um Karriere- und Gehaltseinbußen bei der Frau, andererseits um mangelnde Einbindung in das Familienleben beim Mann (vgl. Astrachan 1992; vgl. Gesterkamp 2005).

In die Karriere und Fähigkeiten von Frauen und Männern zu investieren, ist eine Investition in deren Wohlbefinden, in die Gleichberechtigung und kommt zusätzlich der nächsten Generation zugute. Frauen, wie auch Männer, arbeiten gewissenhaft, sind verlässlich; Mütter, wie auch Väter, haben die Motivation, ihre Kinder mit bestmöglicher Pflege und 
finanzieller Unterstützung zu versorgen, und werden deshalb gute und verlässliche Arbeitnehmerinnen sein (vgl. Berg et al. 2003). Durch die Globalisierung weichen die traditionellen Bedingungen am Arbeitsplatz auf und öffnen die Möglichkeit von Flexibilität. Nicht nur Frauen, sondern auch Männer müssen bei der Erziehung der nächsten Generation, bei gleichzeitiger Ausübung der Erwerbsarbeit, unterstützt werden. Wir geben für diese Ziele folgende Handlungsempfehlungen (s. Abb. 6):

\section{Abb. 6: Handlungsempfehlungen}

\begin{tabular}{|c|c|}
\hline $\begin{array}{l}\text { Gesellschaftliche Erwartung: } \\
\text { Frauen sind oft Hausfrauen und sorgen } \\
\text { für ihre Familie und Kinder; } \\
\text { Männer sind oft Ernährer der Familie, } \\
\text { sorgen für den Unterhalt, die finanzielle } \\
\text { Absicherung }\end{array}$ & $\begin{array}{l}\text { Erziehung des Kindes durch } \\
\text { Väter, Mütter und } \\
\text { gesellschaftliche Institutionen: } \\
\text { Neue politische Richtlinien; } \\
\text { Kinderbetreuungseinrichtungen }\end{array}$ \\
\hline $\begin{array}{l}\text { Diskriminierung: } \\
\text { Frauen werden oft zuletzt eingestellt, } \\
\text { zuerst entlassen, zuletzt befördert und } \\
\text { in weniger prestigeträchtigen Positionen } \\
\text { und Berufen eingesetzt; } \\
\text { Männer werden bevorzugt eingestellt, } \\
\text { befördert, in prestigeträchtige Berufe } \\
\text { eingestellt und zuletzt entlassen }\end{array}$ & $\begin{array}{l}\text { Veränderung des Rollenkonzepts: } \\
\text { Hausarbeit und Kindererziehung } \\
\text { würdigen; } \\
\text { Frauen bestärken, technische Berufe } \\
\text { auszuüben; } \\
\text { Männer bekräftigen, Pflegeberufe } \\
\text { ausüben zu wollen }\end{array}$ \\
\hline $\begin{array}{l}\text { Arbeitgebererwartung: } \\
\text { Arbeitgeber erwarten von Frauen, dass } \\
\text { sie weniger Erfahrung oder Engagement } \\
\text { für den Beruf mitbringen; } \\
\text { Arbeitgeber nehmen an, dass Männer } \\
\text { frei von allen "Lasten und Verpflichtungen“ } \\
\text { sind, die für die Karriere hinderlich sein } \\
\text { können }\end{array}$ & $\begin{array}{l}\text { Kulturelle und normative Änderungen: } \\
\text { Gute Ausbildung für Frauen; } \\
\text { weibliche Vorbilder in Führungspositionen; } \\
\text { mehr Unternehmen, die Belastung durch } \\
\text { Familienarbeit für alle Mitarbeiter } \\
\text { einplanen; } \\
\text { Befreiung der Frauen von der } \\
\text { „Doppelbelastung“ }\end{array}$ \\
\hline
\end{tabular}

Im Europa der Zukunft muss Frauen die Möglichkeit für Karriere und Beruf geboten und Männern eine stärkere Einbindung in das Familienleben ermöglicht werden. Die erste und wichtigste Empfehlung ist gleichzeitig eine Forderung, und zwar nach einer Veränderung der Geschlechterrollenkonzepte von Mann und Frau durch Bildung und Sozialisation. Normen, Werte und Erwartungen an Geschlechterrollen müssen sich ändern, sowohl für die häusliche Arbeit als auch für die Erwerbsarbeit. Dies hat zur Folge, dass eine Umstrukturierung der häuslichen Arbeitsteilung und Kindererziehung möglich werden kann. Die Umstrukturierung der Geschlechterrollenzuschreibungen ist notwendig, damit die Vereinbarkeitsproblematik von Beruf und Familie für Mann und Frau aufgelöst oder zumindest verringert wird. Nicht nur Frauen sind von dieser Problematik betroffen, sondern auch Männer. Frauen möchten ihre 
Karriere nicht aufgeben und trotz Mutterschaft berufstätig sein; Männer möchten stärker in die Familie eingebunden sein und den Fokus ihres Lebens nicht allein auf die Erwerbsarbeit richten (vgl. Mühling/Rost 2007). Das Vorgehen Schwedens bei der Vereinbarkeitsproblematik hat u.a. gezeigt, dass Eltern, die sich die Kindererziehung und Hausarbeit teilen, ein neues und besseres Verständnis für die Unterschiedlichkeit von Verantwortlichkeiten von Männern und Frauen entwickeln und dadurch der Respekt für die häuslichen und familiären Tätigkeiten steigt, da Menschen dazu neigen, Empathie und Mitgefühl zu entwickeln, wenn sie dieselben Rollenerfahrungen gemacht haben (vgl. Hofmeister/Blossfeld 2006). Männer, die die Verantwortung für Kindererziehung und Hausarbeit in der Vergangenheit übernommen haben, sollten in der Lage sein und auch den Willen haben, Arbeitsplatzstrukturen so zu verändern, dass Bedürfnisse von Müttern und Vätern berücksichtigt werden. Wenn Männer und Frauen einen ähnlichen Lebensverlauf aufweisen, das heißt, wenn Männer und Frauen in gewisser Weise gleichermaßen von der Ausführung häuslicher und familiärer Tätigkeiten betroffen sind, dann wird die Möglichkeit für Empathie und Kooperation steigen und eine strukturelle Geringschätzung von Frauen bezüglich der Erwerbsarbeit sinken. Dies hätte zur Folge, dass individuelle Talente unabhängig vom Geschlecht gefördert werden könnten (vgl. ebd.). Es ist folglich wichtig, Stereotype aufzugeben und individuelle Entscheidungen zu unterstützen (vgl. Europäische Kommission 2008).

Die zweite Empfehlung, die sich unmittelbar an die vorherige anschließt, zielt darauf ab, die Pflege von Familienmitgliedern und die Durchführung von häuslicher Arbeit wertzuschätzen. Die fehlende Wertschätzung von Pflegeleistungen stellt ein großes Konfliktpotential in Bezug auf die Vereinbarkeit von Beruf und Familie dar (vgl. Hofmeister/Blossfeld 2006). Erziehung und Pflege von Kindern wird z.B. nicht in das Brutto-Sozialprodukt eingerechnet, obwohl diese Arbeit für das Fortbestehen einer jeden Gesellschaft zentral ist. Unbezahlte Hausarbeit wird als ein selbstverständliches Opfer einer Frau für die Wohlfahrt der Gesellschaft angesehen. Dieses Opfer unbezahlter Arbeit wird von keiner anderen Gruppe in unserer Gesellschaft eingefordert. Dadurch dass sich die Optionen in Bezug auf die Erwerbsarbeit für Frauen im Laufe der Zeit zum Positiven verändert haben und es zu einer Integration der Frau in den Arbeitsmarkt gekommen ist, entscheiden sich mehr Frauen als zuvor gegen die unbezahlte, nicht geachtete Hausarbeit und Pflege von Familienmitgliedern, - was eine sinkende Geburtenrate in vielen europäischen Ländern zur Folge hat (vgl. Olah 1996; vgl. Brewster/Rindfuss 2000). Die Menschen, die für häusliche Arbeit bezahlt werden (z.B. Haushaltshilfen, Tagesmütter), erhalten ein niedriges Gehalt, das dem 
Wert dieser Tätigkeit nicht gerecht wird. Der geringe Lohn lässt sich einerseits auf die Geringschätzung der häuslichen und familiären Arbeit, andererseits auf die private Finanzierung der Hausarbeit zurückführen (vgl. Hofmeister/Blossfeld 2006; vgl. Beck-Gernsheim in diesem Band). Nur wenige Haushalte sind in der Lage, hohe Summen für Kindererziehung und Hausarbeit zu zahlen. Erwerbstätige Frauen, die im Durchschnitt weniger Gehalt als Männer beziehen, sind folglich gezwungen, andere Frauen ,,auszubeuten“, indem sie ihnen weniger Gehalt für Haushaltstätigkeiten zahlen (vgl. ebd.). In vielen Staaten der Europäischen Union übernehmen in großer Zahl ausländische Frauen aus Schwellenund Entwicklungsländern Hausarbeit, Kindererziehung und Pflegeleistungen und müssen daher aufgrund der räumlichen Trennung oft die Versorgung eigener Kinder noch schlechter bezahlten Frauen oder Verwandten überlassen, was sich vor allem auf die Kinder der sozial schwächsten Familie in dieser Kette benachteiligend auswirkt (vgl. Ehrenreich/Hochschild 2004). Wenn dies zur Folge hat, dass die unterbezahlten Pflege- und Haushaltskräfte die Erziehung ihrer eigenen Kinder vernachlässigen müssen, dann ist dieser Kreislauf fehlerhaft und muss verändert und umstrukturiert werden. Wenn ein System geschaffen werden könnte, das auf der Einstellung basiert, dass alle Erwachsenen einmal Kinder waren, eine Erziehung genossen haben, deshalb in Bezug auf Kindererziehung und -betreuung kooperieren und nicht die einzelne Familie mit diesen Aufgaben belasten sollten, dann könnte Ungleichheit, die auf Geschlecht und Klassenzugehörigkeit basiert, transformiert werden (vgl. Hofmeister/Blossfeld 2006).

Unsere Empfehlungen lauten folglich: (1) Hausarbeit, Kindererziehung und Pflege von Familienmitgliedern soll wertgeschätzt und entsprechend entlohnt werden. Wir glauben, dass die gesamte Gesellschaft für alle Kinder verantwortlich ist. Die Gesellschaft trägt letztlich die „Kosten“, die durch eine fehlende oder schlechte Erziehung entstehen, da die Auswirkungen die gesamte Gesellschaft betreffen. Schlechte schulische Leistungen, daraus resultierende schlechte Berufs- und Karrierechancen, und auch ansteigende Kriminalität sind nur einige der Folgen, die die ganze Gesellschaft betreffen. (2) Väter, die sich um ihre Kinder kümmern möchten, sollten gefördert und in ihrem Vorhaben bestärkt werden.

Unsere nächste Empfehlung betrifft die Seite der Arbeitgeber. Arbeitgeber sollen zukünftig familienorientierter sein (vgl. Goedicke/Brose 2008). Ein familienfreundlicher Arbeitgeber sollte seinem Arbeitnehmer (1) flexible Arbeitszeiten und Arbeitsvolumen (vgl. Kramer et al. 1998) und (2) Kinderbetreuungsmöglichkeiten, vielleicht in einem Betriebskindergarten, anbieten. (3) Sozialleistungen für Angestellte mit Kindern 
wie beispielsweise Werkswohnungen, Einkaufsdienste, Familiendarlehen usw. sollten verstärkt angeboten werden. Wenn die Arbeitnehmer autonomer sind, haben sie die Möglichkeit, in das Familienleben eingebunden zu sein und trotzdem einen Beruf auszuüben. Sie werden folglich mit ihrem Leben und Beruf zufriedener sein (vgl. Schilling 2008; vgl. Booth/Ours 2007). Und zufriedene Arbeitnehmer sind in der Regel motiviert, leistungsorientiert und loyal. All dies sind Eigenschaften, von denen der Arbeitgeber profitieren kann und wird (vgl. ebd.). Familienfreundliche Unternehmen sollten in Zukunft keine Minderheit darstellen, da aufgrund der Wirtschaftslage das Zwei-Verdiener-Modell zur Norm werden wird und es deshalb auch möglich sein muss, Beruf und Familie zu vereinbaren.

Ferner sollte die Arbeitsleistung nicht länger mit einer „Anwesenheitskultur" gleichgesetzt werden (vgl. Döge 2004). Hinter dem Konzept der „Anwesenheitskultur“ verbirgt sich ein spezifisches Verständnis von Erwerbsarbeit, das eng an das vorherrschende Rollenverständnis des Mannes als Alleinverdiener und Ernährer geknüpft ist (vgl. ebd.). Wie bereits zuvor erwähnt, wird nach diesem Rollenverständnis nur bezahlte Erwerbsarbeit als Arbeit akzeptiert, Kindererziehung und Hausarbeit stellen nach dem derzeitigen gesellschaftlichen Norm- und Wertesystem keine „Arbeit“ dar (vgl. Neusüss 1992). Eine Umstrukturierung der Betriebskultur führt zu einer kritischen Betrachtung traditioneller Rollenvorstellungen von Mann und Frau und dadurch auch zu einer kritischen Reflexion von bezahlter und unbezahlter Arbeit selbst (vgl. Döge 2004).

Eine weitere Empfehlung ist, dass Müttern und Vätern der Wiedereintritt in das Berufsleben nach dem Erziehungsurlaub erleichtert werden sollte. Das Gehalt von Müttern und Vätern kommt der Gesundheit und Ausbildung ihrer Kinder zugute und bietet zusätzliche Steuereinnahmen für Gesellschaften, denen ein Wohlfahrtsstaat zugrunde liegt. Das Gehalt hilft zudem, für die Absicherung des Unterhalts im Alter zu sparen (vgl. Stegmann 2005). Da Frauen ebenfalls eine Vorbildfunktion für ihre Kinder haben, wird ihnen auf diese Weise ein weiteres Modell des Erwachsenseins vorgelebt, das arbeitende Mütter und Väter als „,normal“ begreift. Mütter und Väter, die wieder in das Berufsleben eintreten, können ihre neu erworbenen Fähigkeiten für ihre weitere Karriere nutzen.

Im 21. Jahrhundert setzt beruflicher Erfolg die Fähigkeit voraus, Ambiguitäten zu bewältigen, verschiedene Tätigkeiten simultan auszuüben und neue Netzwerke von Unterstützung am Arbeitsplatz und im Umfeld zu schaffen. Männer und Frauen müssen in der Kombination von Beruf, Familie und anderen Verpflichtungen erfahren sein. Es scheint, dass Frauen diese Dinge zurzeit besser miteinander vereinbaren 
können (vgl. Sayer 2007). Für Arbeitgeber kann es sich daher lohnen, in weibliche Führungskräfte zu investieren und ein Arbeitsumfeld zu schaffen, in dem Frauen ihre Fähigkeiten nutzen und anwenden können. Berufstätige Frauen - vor allem Mütter - würden von einem familienfreundlichen Unternehmen profitieren (vgl. Peuckert 2008). Diese könnten dazu beitragen, dass Frauen nicht, wie in der Vergangenheit, von Karriere- und Gehaltseinbußen betroffen sind und dass die Entscheidung für die Mutterschaft folglich nicht mehr mit einer impliziten Sanktion einhergeht. Die Wahrscheinlichkeit ist höher, dass Mütter in diesen Unternehmen in ihrer beruflichen Position bleiben und dadurch zu Vorbildern für junge Frauen werden können, die sich eine Vereinbarkeit von Beruf und Familie wünschen (vgl. Krumpholz 2004). Familienfreundliche Unternehmen sollten jedoch nicht nur Rücksicht auf Frauen nehmen und diese fördern, sondern ebenfalls die Bedürfnisse von jungen Vätern berücksichtigen. Diese hegen ebenfalls den Wunsch, in die Familie integriert zu sein und ihr Lebensziel nicht ausschließlich auf die Erwerbsarbeit zu lenken. Eine Integration von Beruf und Privatleben scheint unabdingbar für die Zukunft Europas (s. Abb. 7).

\section{Abb. 7: Lösungsvorschläge}

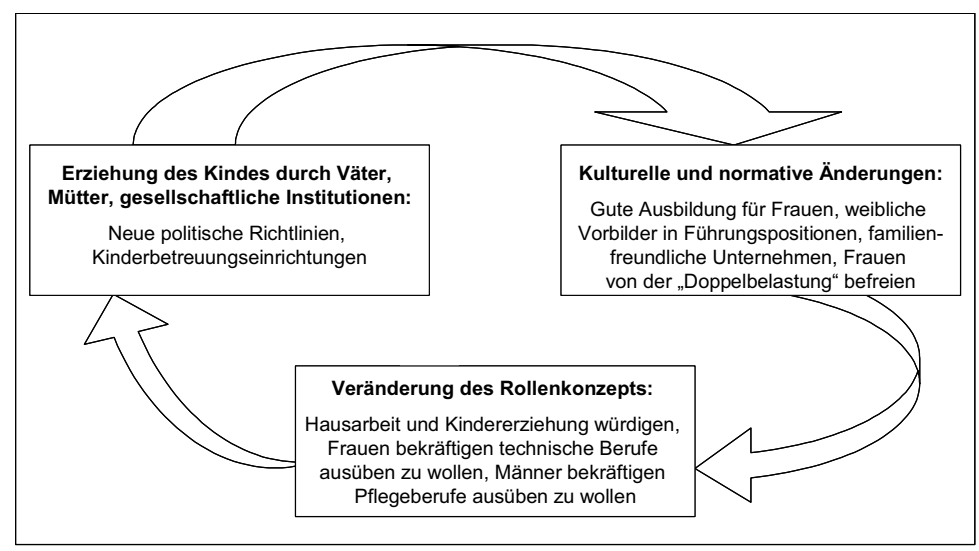

\section{Literatur}

Arn, Christof/Wolfgang Walter (2004): Wer leistet die andere Hälfte der Arbeit? Die Beteiligung von Männern an der Hausarbeit als Bedingung eines „,integralen“ Modells der Zwei-Verdiener-Familie, in: Sigrid Leitner/Ilona Ostner/Margit Schratzenstaller (Hg.): Wohl- 
fahrtsstaat und Geschlechterverhältnis im Umbruch: Was kommt nach dem Ernährermodell? Wiesbaden, S.132-155.

Astrachan, Anthony (1992): Wie Männer fühlen, München.

Baur, Nina (2001): Soziologische und ökonomische Theorien der Erwerbsarbeit, Frankfurt/Main.

Baur, Nina/Heather Hofmeister (2008): Some Like Them Hot: How Germans Construct Male Attractivenes, in: Journal of Men's Studies, Jg. 16, H. 3, S. 280-300.

Becker, Gary S. (1971): The Economics of Discrimination, Chicago.

Berg, Peter/Arne L. Kalleberg/Eileen Appelbaum (2003): Balancing Work and Family: The Role of High-Commitment Environments, in: Industrial Relations, Jg. 42, H. 2, S.168-188.

Bianchi, Suzanne M./Melissa A. Milkie/Liana C. Sayer C./John P. Robinson (2000): Is anyone doing the housework? Trends in the gender division of household labour, in: Social Forces 79, S. 191-228.

Bielby, William T./James N. Baron (1986): Men and Women at Work: Sex Segregation and Statistical Discrimination, in: American Journal of Sociology 91, S. 759-799.

Blau, Francine D. (1984): Discrimination against Women: Theory and Evidence, in: William Darity (Hg.): Labour Economics: Modern Views, Bosten, S. 53-89.

Bolzendahl, Catherine I./Daniel J. Myers (2004): Feminist attitudes and support for gender equality: Opinion change in women and men, 1974-1998, in: Social Forces 83, S. 759-789.

Booth, Alison L./Jan C. van Ours (2007): Job satisfaction and family happiness.The part-time work puzzle, in: Discussion Paper, Forschungsinstitut zur Zukunft der Arbeit GmbH, 3020, Bonn.

Brayfield, April (1995): Juggling Jobs and Kids: The Impact of Employment Schedules on Fathers' Caring for Children, in: Journal of Marriage and the Family, Jg. 57, H. 2, S. 321-332.

Brewster, Karin L./Ronald R. Rindfuss (2000): Fertility and Women's Employment in Industrialized Nations. Annual Review of Sociology 26, S. 271-296.

Brüderl, Leokadia (1992): Frauenleben zwischen Beruf und Familie, Weinheim.

Campbell Clark, Sue (2000): Work/family border theory. A new theory of work/family balance, in: Human Relations 53, S. 747-770.

Cohen, Phillip N. (1998): Replacing housework in the service economy: Gender, Class, and race-ethnicity in service spendine, in: Gender \& Society 12, S. 219-231. 
Coltrane, Scott (2000): Research on household labour: Modeling and measuring the social embeddedness of routine family work, in: Journal of Marriage and the Family 62, S. 1208-1233.

Connell, Robert William (1999): Der gemachte Mann. Konstruktion und Krise von Männlichkeiten, Opladen.

Coontz, Stephanie (2005): Marriage, a History. From Obedience to Intimacy or How Love Conquered Marriage, New York.

Crittenden, Ann (2001): The Price of Motherhood. Why the Most Important Job in the World Is Still the Least Valued, New York.

Degler, Carl N. (1980). At Odds: Women and the Family in America from the Revolution to the Present, Oxford.

Döge, Peter (2004): Auch Männer haben ein Vereinbarkeitsproblem. Familienorientierte Männer im betrieblichen Kontext, online unter: http://gender.verdi.de/genderservice/literatur/lit_maenner, 8.12.08.

Eagly, Alice Hendrickson (1987): Sex differences in social behavior: A social role interpretation, Hillsdale.

Edwards, Jeffrey R./Nancy P. Rothbard (2000): Mechanisms linking work and family: Clarifying the relationship between work and family constructs, in: Academy of Management Review 25, S. 168-184.

Ehrenreich, Barbara/Arlie R. Hochschild (2002): Global Woman. Nannies, Maids, and Sex Workers in the New Economy, New York.

Europäische Kommission (2008): Bericht der Kommission an den Rat, das Europäische Parlament, den Europäischen Wirtschafts- und Sozialausschuss und den Ausschuss der Regionen zur Gleichstellung von Frauen und Männern - 2008, online unter: http://ec.europa.eu/employment_social/gender_equality/docs/com_2008_0010_de.pdf., 9.12.08.

Folbre, Nancy/Julie A. Nelson (2000): For love or money - or both? in: Journal of Economic perspectives 14, S. 123-140.

Folbre, Nancy/Marjorie Abel (1989): Women's work and women's households: Gender bias in the U.S. census, in: Social Research, Jg. 56, H. 3, S. 545-69.

Friedman, Stewart D./Jeffrey H. Greenhaus (2000): Work and Family Allies or Enemies? What happens when business professionals confront life choices, Oxford.

Fux, Beat (2002): Which Models of the Family are Encouraged or Discouraged by Different Family Policies, in: Franz-Xaver Kaufmann/Anton Kuijsten/Hans-Joachim Schulze/Klaus Peter Strohmeier (Hg.): Family Life and Family Policies in Europe, Oxford, S. 363411.

Gershuny, Jonathan (1996): Veränderungen bei der Arbeitsteilung im Haushalt: Mikro-soziologische Analysen, in: Wolfgang Zapf/Jürgen 
Schupp/Roland Habich (Hg.): Lebenslagen im Wandel, Frankfurt/Main, S. 97-124.

Gesterkamp, Thomas (2005): Betriebliche und politische Hindernisse engagierter Vaterschaft, in: Zeitschrift für Familienforschung 1, S. 66-75.

Goedicke, Anne/Hanns-Georg Brose (2008): The Proof of the Pudding is in the Eating: Was heiß „Familienfreundlichkeit“ von Personalpolitik? in: Marc Szydlik (Hg.): Flexibilisierung - Folgen für Arbeit und Familie, Wiesbaden, S. 170-192.

Goldin, Claudia (2004): The Long Road to the Fast Track: Career and Family, in: The ANNALS of the American Academy of Political and Social Science 596, S. 20-35.

Gräfinger, Elisabeth (2001): Die Welt von innen - Männer in Karenz, Unveröffentlichte Diplomarbeit, Wien.

Greenstein, Theodore N. (1996): Husband's Participation in domestic labour: interactive effects of wives' and husbands' gender ideologies, in: Journal of Marriage and the Family 58, S. 585-595.

Hall, Douglas T. (1976): Careers in Organizations, Santa Monica.

Hendrix, Scott (1995): Masculinity and Patriarchy in Reformation Germany, in: Journal of the History of Ideas 56, 2, S. 177-193.

Hersch, Joni/Lesile S. Stratton (2002): Housework and Wages, in: Journal of Human Resources 37, S. 217-229.

Hochschild, Arlie (1989): The Second Shift, New York.

Hofäcker, Dirk (2006): Women's Employment in Times of Globalization: A Comparative Overview, in: Hans-Peter Blossfeld/Heather Hofmeister (Hg.): Globalization, Uncertainty and Women's Careers: An International Comparison, Cheltenham, S. 32-58.

Hofmeister, Heather/Hans-Peter Blossfeld (2006): Women's careers in an era of uncertainty: conclusions from a 13-country international comparison, in: Hans-Peter Blossfeld/Heather Hofmeister (Hg.): Globalization, Uncertainty and Women's Careers. An International Comparison, Cheltenham, S. 433-450.

Hofmeister, Heather/Hans-Peter Blossfeld/Melinda Mills (2006): Globalization, uncertainty and women's mid-career life courses: a theoretical framework, in: Hans-Peter Blossfeld/Heather Hofmeister (Hg.): Globalization, Uncertainty and Women's Careers. An International Comparison, Cheltenham, S. 3-31.

Jürgens, Kerstin/Günter Voß (2007): Gesellschaftliche Arbeitsteilung als Leistung der Person, in: Aus Politik und Zeitgeschichte, H. 34, S. 3-9. 
Kaufmann, Gayle/Peter Uhlenberg (2000): The Influence of Parenthood on the work effort of married men and women, in: Social Forces 78, S. 931-949.

Kimmel, Michael (1987): Changing men: new directions in research on men and masculinity, London.

Kocka, Jürgen (1990): Arbeitsverhältnisse und Arbeiterexistenzen. Grundlagen der Klassenbildung im 19. Jahrhundert, Bonn.

Kolinski, Eva (1995): Women after „Muttipolitik“, in: dies. (Hg.): Between hope and fear. Everyday life in Post-Unification East Germany. A Case Study of Leipzig, S. 177-200.

Kramer, Ulrich (1998): Wettbewerbsstärke und bessere Vereinbarkeit von Familie und Beruf - kein Widerspruch: Flexible Arbeitszeiten in Klein- und Mittelbetrieben, Stuttgart.

Krumpholz, Doris (2004): Einsame Spitze. Frauen in Organisationen, Wiesbaden.

Lachmann, Richard (1995): The Encyclopedic dictionary of sociology, Guilford.

Lück, Detlev (2006): The impact of gender role attitudes on women's life courses, in: Hans-Peter Blossfeld/Heather Hofmeister (Hg.): Globalization, Uncertainty and Women's Careers. An International Comparison, Cheltenham, S. 405-432.

Marx, Karl (1867-94): Das Kapital. Kritik der politischen Ökonomie, Hamburg, in: Marx-Engels-Werke, Bd. 23-25, Berlin 1962-64.

Mills, Charles Wright (1956): The Power Elite, New York.

Mitterauer, Michael (1990): Geschlechtsspezifische Arbeitsteilung in vorindustrieller Zeit, in: Michael Mitterauer (Hg.): Historisch-Anthropologische Familienforschung, Wien, S. 289-314.

Moen, Phyllis (1992): Women's two roles: A contemporary dilemma, New York.

Moen, Phyllis (2001): The Career Quandary, Washington D.C.

Mühling, Tanja/Harald Rost (2007): Väter im Blickpunkt. Perspektiven der Familienforschung, Opladen.

Neusüss, Christel (1992): Die Kopfgeburten der Arbeiterbewegung oder die Genossin Luxemburg bringt alles durcheinander, Osnabrück.

Niehuss, Merith (1999): Die Hausfrau, in: Ute Frevert/Heinz-Gerhard Haupt (Hg.): Der Mensch des 20. Jahrhunderts, Frankfurt/Main, New York, S. 45-65.

Notz, Gisela (1991): „Du bist als Frau um einiges mehr gebunden als der Mann.“ Die Auswirkungen der Geburt des ersten Kindes auf die Lebens- und Arbeitsplanung von Müttern und Vätern, Bonn. 
OECD (2008): OECD Employment Outlook - 2008 Edition. Summary in German, online unter: www.oecd.org/dataoecd/7/52/40938563.pdf, 9.12.08.

Olah, Livia S. (1996): The impact of public policies on the second-birth rates in Sweden: a gender perspective, in: Stockholm Research Reports in Demography, Nr. 98, Stockholm.

Orloff, Ann S. (2002): Women's Employment and Welfare Regimes: Globalization, Export Orientation and Social Policy in Europe and North America, in: Social Policy and Development Programme, Geneva.

Ostner, Ilona (1993): Slow Motion: Women, Work and the Family in Germany, in: Jane Lewis (Hg.): Women and Social Policies in Europe: Work Family and the State, Aldershot, S. 92-115.

Peinelt-Jordan, Klaus (1999): Männer zwischen Familie und Beruf, München.

Peuckert, Rüdiger (2008): Familienformen im sozialen Wandel, Wiesbaden.

Pfau-Effinger, Birgit (2001): Wandel wohlfahrtsstaatlicher Geschlechterpolitiken im soziokulturellen Kontext, in: Bettina Heintz (Hg.): Kölner Zeitschrift für Soziologie und Sozialpsychologie, Jg. 41, Sonderband ,Geschlechtersoziologie', S. 488-511.

Pfau-Effinger, Birgit (2003): Changing Welfare States and Labour Markets in the Context of European Gender Arrangements, online unter: www.socsci.auc.dk/cost/gender/Workingpapers/pfaueffinger.pdf, 8.12.08.

Pfau-Effinger, Birgit (2004): Development of Culture, Welfare States and Women's Employment in Europe, Hants.

Puchert, Ralf/Stephan Höyng (2008): Männer als Akteure im Gleichstellungsprozess? online unter: www.puchert.org/wcg/puchert_hoeyng.pdf, 10.12.08.

Quasier-Pohl, Claudia (2001): Deutsche Eltern im interkulturellen Vergleich, in: Horst Nickel/Claudia Quaiser-Pohl (Hg.): Junge Eltern im kulturellen Wandel: Untersuchungen zur Familiengründung im internationalen Vergleich, Weinheim, S. 301-310.

Raley, Sara B./Marybeth J. Mattingly/Suzanne M. Bianchi (2006): How dual are dual-earner couples? Documenting change from 1970-2001, in: Journal of Marriage and the Family 68, S. 11-28.

Raml, Eva Maria (1993): Karriere und Familie, Linz.

Ranson, Gillian (2001): Men at work - Change or no change? - in the era of the „New Father", in: Men and Masculinities, Jg. 4, H. 1, S. 3-26. 
Reichart, Elisabeth (2007): Doppelte Transformation des Ernährermodells? Eine Längsschnittstudie zur Erwerbsarbeitsteilung bei ostund westdeutschen Paaren nach der Geburt des ersten Kindes, Würzburg.

Röhler, Alexander/Johannes Huinink/Anja Steinbach (2000): Hausarbeit in Partnerschaften. Zur Erklärung geschlechtstypischer Arbeitsteilung in nichtehelichen und ehelichen Lebensgemeinschaften, in: Zeitschrift für Familienforschung, Jg. 12, H. 2 , S. 21-53.

Rotundo, E. Anthony (1993): American Manhood: Transformations in Masculinity from the Revolution to the Modern Era, New York.

Sayer, Liana C. (2007): Gender Differences in the Relationship between Long Employee Hours and Multitasking, in: Research in the Sociology of Work, H. 17, S. 403-435.

Schilling, Elisabeth (2008): Projekt „Glückliches Leben“ zum Zusammenhang von Zeitnutzung und Glück bei berufstätigen Eltern, in: Zeitschrift für Arbeitsforschung, Arbeitsgestaltung und Arbeitspolitik, Jg. 17, H. 1, S. 51-65.

Schnack, Dieter/Thomas Gesterkamp (1998): Hauptsache Arbeit? Männer zwischen Beruf und Familie, Reinbek.

Schneewind, Klaus A./Wolfgang Sierwald (1999): Frühe Paar- und Familienentwicklung: Befunde einer fünfjährigen prospektiven Längsschnittstudie, in: Barbara Reichle/Harald Werneck (Hg.): Übergang zur Elternschaft, Stuttgart, S. 149-164.

Schütze, Yvonne (1988): Mutterliebe - Vaterliebe. Elternrollen in der bürgerlichen Familie des 19. Jahrhunderts, in: Ute Frevert (Hg.): Bürgerinnen und Bürger. Geschlechterverhältnisse im 19. Jahrhundert, Göttingen, S. 118-133.

Stegmann, Michael (2005): Der Einfluss der Kindererziehung auf Erwerbstätigkeitsmuster von Frauen und die Auswirkungen auf das Alterseinkommen, in: Deutsche Rentenversicherung, Jg. 60, H. 12, S. 665-691.

Treas, Judith/Eric D. Widmer (2000): Married women's employment over the life course: Attitudes in cross-cultural perspective, in: Social Forces Jg. 78, S. 1409-1436.

Trepp, Anne-Charlott (1996): Männerwelten privat: Vaterschaft im späten 18. und beginnenden 19. Jahrhundert, in: Thomas Kühne (Hg.): Männergeschichte - Geschlechtergeschichte, Frankfurt/Main, New York, S. 31-50.

Vaskovics, Lazlo/Harald Rost (1999): Väter und Erziehungsurlaub, in: Schriftenreihe des Bundesministeriums für Familie, Senioren, Frauen und Jugend, Bd. 179, Stuttgart. 
Volz, Rainer (2007): Väter zwischen Wunsch und Wirklichkeit. Zur Beharrlichkeit traditioneller Geschlechterbilder, in: Tanja Mühling/Harald Rost (Hg.): Väter im Blickpunkt. Perspektiven der Familienforschung, Opladen, Farmington Hills, S. 205-224.

Weber, Max (1976): Wirtschaft und Gesellschaft. Grundriss der verstehenden Soziologie, Tübingen.

Werneck, Harald (1998): Übergang zur Vaterschaft. Auf der Suche nach den „,neuen“ Vätern, Wien.

Williams, Joan (2000): Unbending Gender. Why Family and Work Conflict and What to Do About It, Oxford.

Witt, Nadine (2008): He thinks, she thinks: „Thinking“ Gender in the Household, unveröffentlichte Magisterarbeit.

Yalom, Marilyn (2001): A History of the Wife, New York.

Zook, Melinda S. (2002): „Integrating Men's History into Women's History: A Proposition“, in: The History Teacher, Jg. 35, H. 3, S. 373-387. 